\section{MS39-P14 Trends on multilayer X-ray optics and scatterless pinholes}

Jörg Wiesmann ${ }^{1}$, Frank Hertlein ${ }^{1}$, Andreas Kleine ${ }^{1}$, Carsten Michaelsen ${ }^{1}$, Uwe Heidorn ${ }^{1}$, Christopher Umland ${ }^{1}$, Michael Störmer ${ }^{2}$

1. Incoatec GmbH, Max-Planck-Strasse 2, 21502 Geesthacht, Germany

2. Helmholtzzentrum Geesthacht, Max-Planck-Strasse 1, 21502 Geesthacht, Germany

\section{email: wiesmann@incoatec.de}

A large variety of X-ray optics is required for beam alignment, guidance or monochromator applications at synchrotron beamlines. We investigated various types of multilayer optics for energy ranges between several keV and up to $80 \mathrm{keV}$. For high-brightness laboratory sources like the latest Incoatec Microfocus Source I $\mu$ S or metaljet based sources, optics with similar shape errors are nowadays needed. They are used as monochromators and beam shaping devices in protein and small molecule crystallography, as well as in powder diffraction and small angle scattering.

We will present new developments of 2-dim beam shaping Montel Optics for synchrotron applications and for high-brilliance laboratory equipment. At synchrotrons such as DLS, NSLS and APS, different types of these optics are now used, for example in analyzer systems for inelastic scattering. For the optics a perfect multilayer coating have to be produced with a precision within $\pm 1 \%$ of the single layer spacing. Very low shape errors below $100 \mathrm{~nm}$ and figure errors below 5 arcsec are required for the substrates. In summary, this ensures also for laboratory sources superb flux densities of above $4 \times 10^{11}$ phts $/ \mathrm{s} / \mathrm{mm}^{2}$.

In this contribution we will also present cutting-edge results of a $50 \mathrm{~cm}$ laterally graded multilayer optics, developed for special mini-synchrotrons with a deviation to a specified film shape of less than $0.3 \%$.

Besides the multilayeroptics, other components shape the X-ray beam. For many experimental set-ups, the collimator and pinhole system plays a significant role. Parasitic aperture scattering causes loss in data quality especially in SAXS and GISAXS applications. Various measurement results will be presented showing the improvement of data quality with Incoatec's scatterless pinholes called SCATEX. These pinholes are either made of Germanium for energies $<11.2 \mathrm{keV}$ or of Tantalum for energies $>11.2 \mathrm{keV}$ and are available with diameters from $2 \mathrm{~mm}$ down to $20 \mu \mathrm{m}$ and below.

Keywords: Multilayer Optics; Scatterless Pinholes; Montel Optics; Metaljet; Microfocus Source; Synchrotron Beamline

\section{MS39-P15 Fast and efficient - experiments with modern X-ray sources and CMOS APS detectors}

Holger Ott ${ }^{1}$, Bruce C. Noll ${ }^{2}$

1. Bruker AXS GmbH

2. Bruker AXS Inc.

email: holger.ott@bruker.com

New techniques for experimental design become available with the PHOTON detector's continuous-scans in shutterless mode. Until today, crystal screening has been a time-consuming task that hardly revealed more than the unit-cell dimensions. For one second exposures in shutterless mode, overall data collection times are reduced by as much as $75 \%$ as long overhead times of CCD detectors are eliminated. Modern high-flux X-ray sources also generally lead to shorter exposure times per degree for a given sample. Consequently, the combination of the PHOTON detector with the new $\mathrm{X}$-ray sources allows collecting often complete data sets in as little as $60 \mathrm{~s}$.

These scans provide impressively more reflections for indexing than traditional matrix scans in far less time. The huge amount of information enables the sophisticated analysis of the diffraction pattern, e.g. for multifold twinning or modulation, using the powerful tools available in the newly released APEX3 software suite. Based on this examination, crystal defects are quickly identified, which may suggest the merit of picking another crystal. As an additional benefit, even the structure determination can be performed, revealing the chemical constitution of the crystal. This facilitates an easy decision-making to discontinue or continue the experiment with improved knowledge for an eventually desired more exhaustive data collection.

Typical day to day examples will be presented taking advantage of the latest PHOTON APS detector and X-ray source combinations showing how complete data collections can be finished in the time frame of a traditional pre-experiment.

Keywords: CMOS APS Detectors, Modern X-ray Sources, Fastand Efficient Data Collections 